\title{
A Comparative Study of Testing Parameters of Medical Images Processing
}

\author{
Fatima Qamar ${ }^{1}$ and Mehreen Sirshar ${ }^{2}$ \\ ${ }^{1}$ Fatima Jinnah Women University, the Mall, Rawalpindi, Pakistan \\ ${ }^{2}$ Fatima Jinnah Women University, the Mall, Rawalpindi, Pakistan \\ ${ }^{1}$ fatimaqamar111@gmail.com, ${ }^{2}$ msirshar@gmail.com
}

\begin{abstract}
Medical imaging is a technique or a process that is used in the visualization of body tissues, parts or organs for the usage in clinical analysis, treatment and disease monitoring. As medical imaging is used to reveal the internal structure of body that is hidden by the bones or skin, so there raises a need of a quality of an image that gives an accurate and exact information of the body without any loss of information. So this paper presents the comparative study of testing or the quality parameters of the medical image processing that should be check in order to extract the exact information of an image.
\end{abstract}

Keywords: Medical image processing, testing, quality parameters

\section{Introduction}

Medical Image processing is a process of the creation of visual representations of the internal structure of a body, along with the visual demonstration of some organs or tissues function. The main usage of medical image processing is to reveal the internal structures that are hidden by the bones or the skin of body, that are furtherly used to diagnose and treat the disease.

There are many techniques of medical imaging that can be used as a non-aggressive procedures of viewing inside the body. That helps the medical practitioners to don't opened the body of patient surgically to look at several organs and areas. That also assist the medical practitioners to diagnose or treat the different medical conditions of the patient.

As a discipline, it is part of biological imaging and integrates radiology which uses the imaging technologies of magnetic resonance imaging, endoscopy, $\mathrm{X}$ ray radiography, medical ultrasonography or ultrasound, thermography, tactile imaging, (SPECT) Single-photon emission computed tomography medical photography, electrography, and (PET) positron emission tomography.

As the medical image processing is used in many critical fields that have a main purpose to deal with patient's health. This concept arises the importance of testing or the quality parameters to validate the quality of an image. The high quality imaging growing demand has been increasing day by day not only in software but also in hardware technology. Nowadays, the hardware designer try their best in evolving best sensors, best optics, or most proficient design for having great image quality. In order to produce high quality image, this paper presents the testing or quality parameters to validate the accuracy and the correctness of an image.

In this paper the literature review of medical image processing is done in second section. Analysis on these papers is done in section three. Conclusion is given in fourth section. 


\section{Literature Review}

\section{[1]Gabor Barcodes for Medical Image Retrieval}

Gabor transform is used in this paper to extract texture-based data of medical images. The important property of Gabor features is its strength of measuring of translation, scale, rotation, and photometric turbulences, like noise or illumination changes. As an innovative framework for the images annotation, this paper used Gabor Barcodes (GBCs). To classify GBC for a given image which results in varied retrieval performances along with varied barcode lengths, the different parameters to check the effects of Gabor filters, i.e., diverse sets of orientations and scale is applied. IRMA dataset is used to evaluate the Content-based image retrieval performance. The $80 \%$ accuracy was achieved in image retrieval by using Gabor Barcodes.

\section{[2]Metrology Applications Of Quantitative Evaluation methods In Ct Phantom Images}

In this paper, for the Catphan500 phantom images standardization, a time-efficient and quantitative program method is used, also known as an automated program mode. The main purpose of the usage of automated program mode is the calibration of main parameters including CT number, image uniformity, and high spiral resolution along low contrast resolution parameter. The estimation of manual subject to the evaluation of automatic objective transition is the main purpose of this program mode, and to improve the work efficiency as well. It will be made more efficient by applying this program mode in the MTF method. In the future research, more optimized and enhanced methods will be applied.

\section{[2]Level Set Segmentation with Shape Prior Knowledge Using Intrinsic Rotation, Translation and Scaling Alignment.}

For medical image segmentation this paper presents the Level set-based algorithms have been widely used. For rotations, translation, and scaling invariance, the restriction of shape needs to be integrate. Optimal registration parameters finding during the curve evolution is the main approach to solve a registration problem of an image. The paper extends the work of Cremers in shape prior knowledge who proposed the translation alignment and intrinsic scaling to measure shape dissimilarities. In this paper, a complete equations set was derived for the intrinsic rotation comprising in the presented framework. To use shape priors in complex segmentation problems, the evolution equation incorporated with covariance matrix like eigenvector and eigenvalues matrices of each shape is used. Synthetic images and 2D right ventricle MRI images are used to test the combination of this algorithm and Chan-Vese function.

\section{[3]Performance Comparison of Discrete Orthonormal S-Transform for the Reconstruction of Medical Images}

This paper examines the performance of the (DOST) Discrete Orthonormal STransform technique for medical images reconstruction. This technique is compared with the Discrete Wavelet Transform (DWT) and q-Recursive Zernike Moment (q-RZM) on the basis of measurement of performance on medical images, namely Mean Square Error (MSE) or Peak Signal to Noise Ratio (PSNR). Images of Magnetic Resonance (MR) along the Computer Tomography (CT) are used to test the feasibility of the techniques to be used for image compression. DOST should be considered for more investigation of the compression processes as medical image compression needs lossless information of the reconstructed image. The consequence of decoding and encoding are the vital processes of image compression before the image is finally reconstructed. From the results of 
experiment, the DOST technique has high PSNR value and outperforms qrecursive Zernike Moments and Discrete Wavelet Transform Techniques.

\section{[4]The Effect of Region of Interest Size on Textural Parameters}

This paper focuses on the images quantitative data regarding the properties of an image that is mainly done by Texture analysis. To ensure proper image normalization, this paper emphases on more than one pixel relation in texture parameters. As it seems that lots of parameters depend on the ROI size, but the practice for region of interest (ROI) definition in the texture parameters does not exist. The ROI size effect of artificial noise images and magnetic resonance head images was studied. The great effect of ROI size has examined on the numerous second order texture features calculated result. So the falsely classification of analyzed tissues can be resulted from different size ROIs comparisons, as every application has its own specific ROI size.

\section{[6]A preliminary study on a method for objective uniformity assessment in diagnostic ultrasound imaging.}

For failures detection of shapes uniformity, in some cases an ultrasound image uniformity assessment is used to examine the quality of an image. This paper presents a novel method that is used to evaluate B-mode type of image uniformity on the specific area. To detect non uniformities of an image, the specified method is applied that centered on the gray level histogram of image along influenced via a sigmoid function (SWHM). To examine the uniformity of an image according to specified rectangular ROIs size, SWHM is bound to proliferate the result's robustness. This method splits the index of uniformity in two measures including UROIx a transversal uniformity index and a longitudinal uniformity index UROIz. Since preliminary results look encouraging to refine the method for the improvement of the SWHM, which aims to contribute towards the quality assurance standardization of investigative ultrasound scanners.

\section{[5]Feature Points Based Image Registration between Endoscope Image and the CT Image}

According to MIS robot system's special requirements, this paper presents a fast feature point on the basis of local registration method of medical image. For image similarity, a Euclidean distance is introduced by this algorithm that is used to measure distance between two or more feature points on special tissue and general tissues. To efficiently solve the transformation function of registration, the ICP algorithm framework is used in this paper. This algorithm is tested on the medical images which results in matching the inclusive structure of an image along aligning information of image's anatomical location. This registration method is a robust, accurate and fast method of registering medical images as the results of this registration method are satisfactory.

\section{[8]A New Content-Based Medical Image Retrieval System Based on Wavelet Transform and Multidimensional Wald-Wolfowitz Runs Test}

A new medical image content-based retrieval system on the basis of weighted MWW runs test and wavelet transform (DWT) symlet is presented by this paper. In order to compare two color images, this algorithm centered on the predictable similarity measures. To extract medical images' texture features the DWT is used while the weighted MWW runs test is used to compare texture features distributions of two medical images. The main aim of the suggested algorithm is that it can practice by medical images of different kinds and having varied resolution. The algorithm was executed on almost 1,000 medical images and the results show great efficiency and accuracy in retrieving the medical 
images, along generating highly precision results. The more work will be done in the future relating to medical image which have low precision.

\section{[6]Interoperability Testing Software for Sharing Medical Documents and Images}

In order to share images among different organizations, this paper presents an architecture and the functionalities of a type of web application for evaluating and testing interoperability parameter in field of healthcare. Moreover, this paper describes XDS framework comprising of numerous web technologies. XDS framework is an Electronic Health Record's turning stone that is in positioning of various nations. XDS qualifications is advanced in order to improve the flexibility, efficiency, interoperability, and maintainability in sharing of medical images. System implementers use XDS-I testing software to test their implementations. The site integrators might be use it to validate the interoperability as it can share medical images and the developers can use it to overcome implementations' problems, and to recognize qualifications uncertainties.

\section{[10]An innovative multimodal/ multi spectral image registration method for medical images based on the expectation-maximization algorithm}

This paper presents a methodology for geometric registration of multispectral/multimodal image on the basis of Expectation Maximization (EM) methodology. This methodology estimates the geometric transformation parameters that scales or aligns the multispectral/multimodal images, comprising of two step iterative procedure. On every step of procedure, a novel approximation of the geometric transformation and the joint conditional multispectral intensity distribution is calculated. To relate multispectral intensity values between multimodal images, the intensity relations are linked to the hidden variables between the images in it. By testing the proposed algorithm on varied medical images, which results in better alignment of multispectral/multimodal medical images. An extension of a $3 \mathrm{D}$ version of the multimodal/multispectral registration method is considered as a future work, with an elastic registration based on the same EM algorithm.

\section{[11]Recursive anisotropic diffusion denoising}

This paper presents an image denoising algorithm known as the recursive anisotropic diffusion algorithm. For image smoothing and noise removal a recursive anisotropic filter is used. The better noise removal with similar parameters resulted from the extension of conservative anisotropic diffusion model towards recursive model, which leads to better results in fewer iterations. The improved preservation of edge is correspondingly examined by the reduce numbers of iterations. With fewer iterations objective and visual performance evaluation illustrates great consequences than the classical model. Successful noise deduction with good edge preservation is examined by the testing this algorithm on real medical images.

\section{[12]Secure Transfer of Medical Images Using Hybrid Encryption Authentication, Confidentiality, Integrity}

For message confirmation and message confidentiality using security of transport layer, different symmetric or asymmetric techniques are accomplished for end to end transmission in data Security. In order to secure medical images transmission, this paper presents a new hybrid encryption system, and built on RSA and AES procedures. For data confidentiality AES algorithm is applied while on behalf of authentication RSA is used. The basic correlation function assures integrity among image's adjacent pixels which enables the evaluation of quality of the encryption cryptosystems. As each image 
produces a different cryptographic fingerprint, this encryption system generates a unique password for each image and to verify the integrity of the image.

\section{[13]Medical Imaging in Heterogeneous Telemedicine Network}

The literature of medical imaging in area of heterogeneous telemedicine network is presented in this paper. Under various traffic loads, the testing of the wireless protocols appropriate in wireless local area network and personal area network (PAN) is done on DICOM formatted medical images of MRI, US and CT. For PAN communication, the two Bluetooth protocols are mainly focused including L2CAP and RFCOMM, while server and FTP client are observed in Wi-Fi transmission. For medical applications, the delay transmission time occurrence in image transfer is recorded. In order to reduce the transit time the CS means compressive sensing technique is applied for image size reduction to almost half. By applying L2CAP, the transmission time is smaller than the RFCOMM protocol. The MSE, PSNR and SSIM parameters are used to measure the quality of reconstructed image. Images related to MRI and CT are reassembled having relatively improved quality parameter outcomes while there is a requirement of applying image denoising algorithms for the reconstruction of US images.

\section{[14]Segmentation Methods for Computer Aided Melanoma Detection}

Melanoma is a deadly form of skin cancer, to detect melanoma in the initial stage, image processing techniques helps to build the automated screening system. The segmentation process is an important step in the automated system of melanoma detection which involves border detection to extract the lesion area from the background skin. Adaptive thresholding, statistical region merging, color enhancement, multilevel thresholding, iterative stochastic region merging and iterative segmentation as the segmentation techniques are discussed in this paper. These segmentation methods are compared on the basis of accuracy, sensitivity and specificity parameters. The highest accuracy and specificity is achieved from multilevel thresholding and maximum sensitivity is obtained for iterative stochastic region merging. By including the textural information of the skin lesion, the accuracy of the segmentation algorithms can be further improved.

\section{[15]An Effective Non-rigid Image Registration Method Based on Active Demons Algorithm}

To adjust the driving force combined with homogeneous coefficient, a non-rigid registration algorithm on the basis of active demons algorithm is presented by this paper which leads to balance coefficient parameter. The restriction cause of the registration accuracy and the convergence speed might be reduced to some level along with the small and large distortion. The accuracy of registration and the convergence speed is further improved by introducing a strategy in registration process known as coarse to fine multiresolution strategy. The experiment on natural, medical and test images results in the efficient and accurate method in registration, by showing accuracy close to image registration algorithm of TV-L1 optical flow that solves active demons algorithm problem.

\section{[16]A Matlab Based GUI for Multiframe Super Resolution Reconstruction of Different Gray Scale Images with and without Adaptive filter}

This paper presents a robust technique of Multi frame SRR, in which Lucas Kanade Pyramidal optical flow algorithm is used to developed frame to frame image registration iteratively, in order to remove noise and to produce a great quality super resolution image. To obtain HR image Add method, Median Shift, down sampling, Gaussian filter, gradient 
back projection or gradient regularization is performed. To estimate the quality parameters of image such as SD, SSIM, RMSE, and PSNR, a GUI based on MATLAB is developed for data generated synthetically. The quality parameters of HR image with and without adaptive filters are tested in MATLAB based GUI. The experiment results in image quality improvement, along the maintenance of edge data and better visual effect using adaptive filters.

\section{[17]Generic Method for Intensity Standardization of Medical Images using Multiscale Curvelet Representation}

Using multi-scale curvelet transform, this paper presents a basic method for $2 \mathrm{D} / 3 \mathrm{D}$ medical images' intensity standardization. To measure the discrete curvelet sub bands energies to a reference energy an iterative procedure is used by this method. To calculate a value of reference energy for sub band tracking using the curvelet transform, the reference data are first decomposed into scale and then to localize sub bands in training stage. The scaling of value of each sub band's localized energy to reference localized energy is done in testing stage. To obtain the standardized intensity image having intensity variations preservation, a transform Curvelet is used which allows the independent scaling of individual structures. In pilot study, the projected process effectively results in standardized multi-modal imaging intensities data on CXR and MRI. To improve the accuracy of CAD on data, the proposed method might be implemented as a preprocessing step for 3D and 2D data.

\section{[18]Comparative Analysis between a Variational Method and Wavelet Method PURE-LET to Remove Poisson Noise Corrupting CT images}

Poison noise normally disturbs various images types and especially medical images which rebuilt after an $\mathrm{X}$ radiation. This paper presents two methods for Poisson noise removal. A restoring method that takes into the actual appearance explanation of poison noise known as VAMPN-CT method derived from variational approach. Poisson Unbiased Risk Estimation-Linear Expansion of thresholds known as PURE-LET is derived from wavelet approach. The paper presents the comparison which evaluates the robustness and reliability of either the VAMPN-CT or the PURE-LET results in annoying factors such as spatial resolution, artifact etc., in medical image processing. Different PSNR of a given image in repeating algorithm is examined by PURE-LET method that leads to unstable method. The captivating spatial and contrast resolution resulted from VAMPN-CT that causes to highest PSNR in images of CT. In image quality and in runtime environment, the VAMPN-CT excels at the Pure-Let wavelet method according to the experimental results.

\section{[19]Testing of several CT performance phantoms for medical imaging}

In this paper, under the same clinical circumstances about one hundred CT performance phantoms were verified, in order to examine the Computed Tomography (CT) number's module parameters. This paper presents the CT performance phantoms' measurement of low, high contrast resolution and the image uniformity. In regardless of using the same CT scanner experiment results in the testing disparities values of various CT performance phantoms. To meet the requirements of CT performance test, standard of CT performance phantom must be traceable according to experimental results. For the detection of the phantom CT performance specifications, the requirement of realistic quantifiable indicators for $\mathrm{CT}$ phantom test is raise. 


\section{[20]Tomographic Image Reconstruction Using Filtered Back Projection (FBP) and Algebraic Reconstruction Technique (ART)}

Using Filtered Back Projection (FBP) and Algebraic Reconstruction Technique (ART), the comparisons of the image reconstruction algorithms is presented in this paper. To reconstruct the entity from X-ray projection FBP and ART methods are applied. In order to locate the objects' inner or hidden defects image reconstruction is applied that derives from computed tomography. The reconstructed image quality is measured by four performance parameters i.e., df, dp, MSP an NCC. Two phantoms such as abdomen standard medical image and the head model of Shepp-Logan phantom are used for the projection of image reconstruction. According to the less error values of dp, df, MSP an NCC, FBP technique delivers the best image quality than those of ART. So FBP procedure is practical and reliable in order to improve the reconstructed images quality.

\section{Analysis}

Parameters used are explained in table I. Analysis has been done through table IIa, table IIb and table IIc. Nouredanesh et. al., [1] presents Gabor barcodes having the important property of its strength of measuring of translation, scale, rotation, and photometric turbulences, like noise or illumination changes. Arrieta et. al., [3], Yusof et. al., [4], Sikiö et. al., [5], Scorza et. al., [6], Lokhande et. al., [16], Kais et. al., [18], Chetih et. al., [20] discuss the noise of the image as the important parameter of image processing. For image denoising Ostojić et. al., [11] presents a recursive anisotropic diffusion algorithm.

Zhang et. al., [2] presents the usage of automated program mode is the calibration of main parameters including CT number, image uniformity, and high spiral resolution along low contrast resolution parameter. Arrieta et. al., [3], Scorza et. al., [6], Nakaram et. al., [8], Tiwari et. al., [13], Tang et. al., [15], Lokhande et. al., [16], Kais et. al., [18], discuss the resolution parameter in image processing. Li et. al., [19] presents the CT performance phantoms' measurement of low, high contrast resolution and the image uniformity.

Yusof et. al., [4], Xiao-jun et. al., [7], Tiwari et. al., [13], Kais et. al., [18], Li et. al., [19] consider the CT number as the parameter of the image processing. Zhang et. al., [2] the usage of automated program mode is the calibration of main parameters including CT number, image uniformity, and high spiral resolution along low contrast resolution parameter in a quantitative way.

Zhang et. al., [2], Sikiö et. al., [5] Li et. al., [19] discuss the image uniformity as a system testing parameter. Scorza et. al., [6] presents a novel method that is used to evaluate B-mode type of image uniformity on the specific area.

Nouredanesh et. al., [1] presents Gabor transform having the important property of its strength of measuring of translation, scale, rotation, and photometric turbulences, like noise or illumination changes. Arrieta et. al., [3] propose the translation alignment and intrinsic scaling to measure shape dissimilarities. Xiao-jun et. al., [7], Nakaram et. al., [8], Bérubé et. al., [9], Arce-Santana et. al., [10], Mansoor et. al., [17] consider the transformation parameter i.e., translation, rotation and scaling in image processing as it restricts the shape information.

For image denoising Ostojić et. al., [11] presents a recursive anisotropic diffusion algorithm which shows noise removal along edge preservation. Lokhande et. al., [16] discuss a SRR which maintains the edge information and better visual effect using adaptive filters.

Xiao-jun et. al., [7] presents a novel feature point on the basis of local registration method of medical image. For image similarity, a Euclidean distance is introduced by this algorithm. Nouredanesh et. al., [1], Arrieta et. al., [3], Ostojić et. al., [11], Tiwari et. al., [13], Tang et. al., [15], Mansoor et. al., [17], Chetih et. al., [20] discuss image similarity as the main parameter in image processing. Nakaram et. al., [8] presents a new medical 
image content-based retrieval system on the basis of weighted MWW runs test and wavelet transform (DWT) symlet. In order to compare two color images, this algorithm centered on the predictable similarity measures.

Yusof et. al., [4] examines the performance of the Discrete Orthonormal S-Transform (DOST) technique for the reconstruction of medical images. It measures the performance parameters i.e., PSNR and MSE. Lokhande et. al., [16] estimate the quality parameters of image such as SD, SSIM, RMSE, and PSNR, a GUI based on MATLAB is developed for data generated synthetically. Ostojić et. al., [11], Tang et. al., [15], Kais et. al., [18] examines the performance parameters i.e., PSNR and MSE in image processing.

Yusof et. al., [4] examines the performance of the Discrete Orthonormal S-Transform (DOST) technique for the reconstruction of medical images against performance parameters i.e., PSNR and MSE. Tang et. al., [15], Lokhande et. al., [16], Kais et. al., [18] discuss MSE parameter which is a main parameter in measuring the quality of reconstructed image. Chetih et. al., [20]. Tiwari et. al., [13] discuss the MSE, PSNR and SSIM parameters which are used to measure the quality of reconstructed image.

Ostojić et. al., [11], Tiwari et. al., [13], Tang et. al., [15] examines the quality parameters i.e., PSNR and MSE of image processing. Lokhande et. al., [16] estimate the quality parameters of image such as SD, SSIM, RMSE, and PSNR, a GUI based on MATLAB is developed for data generated synthetically. The experiment results in image quality improvement, along the maintenance of edge data and better visual effect using adaptive filters.

Sikiö et. al., [5] emphases on more than one pixel relation in texture parameters. As it seems that lots of parameters depend on the ROI size. To examine the uniformity of Scorza et. al., [6] an image according to specified rectangular ROIs size, SWHM is bound to proliferate the result's robustness.

Arrieta et. al., [3] propose the translation alignment and intrinsic scaling to measure shape dissimilarities. Xiao-jun et. al., [7] presents a novel feature point on the basis of medical image registration method. This algorithm is tested on the medical images which results in matching the inclusive structure of an image along aligning information of image's anatomical location. Arce-Santana et. al., [10] presents methodology for geometric registration of multispectral/multimodal image on the basis of Expectation Maximization (EM) methodology.

Belkaid et. al., [12] present presents a new hybrid encryption system, and built on RSA and AES procedures, it evaluates whether the image is encrypted or not.

Belkaid et. al., [12] presents the basic correlation function that assures integrity among image's adjacent pixels which enables the evaluation of quality of the encryption cryptosystems.

The accuracy of registration and the convergence speed is further improved by Tang et. al., [15] introducing a strategy in registration process known as coarse to fine multiresolution strategy. Mansoor et. al., [17] discuss multi-scale curvelet transform to improve the accuracy of CAD on data, the proposed method might be implemented as a preprocessing step for 3D and 2D data.

Table I. Evaluation Parameters

\begin{tabular}{|l|l|l|}
\hline $\begin{array}{l}\text { Evaluation } \\
\text { Parameters }\end{array}$ & Definition & $\begin{array}{l}\text { Possible } \\
\text { values }\end{array}$ \\
\hline Image Noise & $\begin{array}{l}\text { Image noise is the pixels standard deviation } \\
\text { in a homogenous image region. }\end{array}$ & Yes/No \\
\hline Resolution & It measure the sharpness of the image. & Yes/No \\
\hline CT number & $\begin{array}{l}\text { In a computed tomogram, it is a calculated x-ray } \\
\text { absorption constant value of a pixel of an image. }\end{array}$ & Yes/No \\
\hline Image uniformity & The CT numbers consistency of the images & Yes/No \\
\hline
\end{tabular}




\begin{tabular}{|l|l|l|}
\hline & over the field of scan. & \\
\hline transformation & $\begin{array}{l}\text { Transformation is a function which maps } \\
\text { one set to another set. It measures whether } \\
\text { the image is transformed by rotation, } \\
\text { scaling and translation }\end{array}$ & $\begin{array}{l}\text { Rotated, } \\
\text { scaled and } \\
\text { translated }\end{array}$ \\
\hline Edge-preservation & $\begin{array}{l}\text { It smooths away textures whilst retaining } \\
\text { sharp edges. }\end{array}$ & Yes/No \\
\hline PSNR & $\begin{array}{l}\text { It is a ratio of maximum power signal and } \\
\text { the corrupting noise power which disturbs } \\
\text { the representation reliability. }\end{array}$ & Yes/No \\
\hline MSE & $\begin{array}{l}\text { MSE is mean of square of errors based on } \\
\text { image intensity scaling. }\end{array}$ & Yes/No \\
\hline SSIM & $\begin{array}{l}\text { SSIM is used for measuring the similarity } \\
\text { between two images. }\end{array}$ & Yes/No \\
\hline ROI Size & $\begin{array}{l}\text { A ROI means region of interest is a portion } \\
\text { of an image you want to filter. The } \\
\text { processing time depends on ROI size. }\end{array}$ & Yes/No \\
\hline Image Alignment & $\begin{array}{l}\text { The alignment of the image element are } \\
\text { used to specify the placement and size of } \\
\text { images when generated to an output } \\
\text { delivery format. }\end{array}$ & Yes/No \\
\hline Image Similarity & $\begin{array}{l}\text { It provide a quantitative measure of the } \\
\text { degree of match between image patches. }\end{array}$ & Yes/No \\
\hline Encryption & $\begin{array}{l}\text { It hides the information of an image to } \\
\text { protect it from unauthorized access. }\end{array}$ & Yes/No \\
\hline Integrity & $\begin{array}{l}\text { It is the quality of being reliable and } \\
\text { trustworthy. }\end{array}$ & Yes/No \\
\hline Accuracy & $\begin{array}{l}\text { It is the true value and the mean value } \\
\text { information. }\end{array}$ & Yes/No process that generates the \\
\hline
\end{tabular}

Table Ila. Analysis of Parameters

\begin{tabular}{|l|l|l|l|l|l|}
\hline References & $\begin{array}{l}\text { Image } \\
\text { Noise }\end{array}$ & Resolution & $\begin{array}{l}\text { CT } \\
\text { number }\end{array}$ & $\begin{array}{l}\text { Image } \\
\text { Uniformity }\end{array}$ & Transformation \\
\hline $\begin{array}{l}\text { Nouredanesh } \\
\text { et. al., }[1]\end{array}$ & Yes & No & No & No & $\begin{array}{l}\text { Rotated, scaled } \\
\text { and translated }\end{array}$ \\
\hline $\begin{array}{l}\text { Zhang } \text { et. al., } \\
{[2]}\end{array}$ & No & Yes & Yes & Yes & No \\
\hline $\begin{array}{l}\text { Arrieta } \text { et. al., } \\
{[3]}\end{array}$ & Yes & Yes & No & No & $\begin{array}{l}\text { Rotated, scaled } \\
\text { and translated }\end{array}$ \\
\hline $\begin{array}{l}\text { Yusof } \text { et. al., } \\
{[4]}\end{array}$ & Yes & No & Yes & No & No \\
\hline $\begin{array}{l}\text { Sikiö } \text { et. al., } \\
{[5]}\end{array}$ & Yes & No & No & Yes & No \\
\hline $\begin{array}{l}\text { Scorza } \text { et. al., } \\
{[6]}\end{array}$ & Yes & Yes & No & Yes & No \\
\hline $\begin{array}{l}\text { Xiao-jun } \text { et. } \\
\text { al., }[7]\end{array}$ & No & No & Yes & No & $\begin{array}{l}\text { Rotated and } \\
\text { translated }\end{array}$ \\
\hline $\begin{array}{l}\text { Nakaram } \text { et. } \\
\text { al., }[8]\end{array}$ & No & Yes & No & No & $\begin{array}{l}\text { Rotated, scaled } \\
\text { and translated }\end{array}$ \\
\hline $\begin{array}{l}\text { Bérubé } \text { et. al., } \\
{[9]}\end{array}$ & No & No & No & No & $\begin{array}{l}\text { Rotated, scaled } \\
\text { and translated }\end{array}$ \\
\hline Arce-Santana & No & No & No & No & Rotated, scaled \\
\hline
\end{tabular}




\begin{tabular}{|l|l|l|l|l|l|}
\hline et. al., $[10]$ & & & & & No \\
\hline $\begin{array}{l}\text { Ostojić } \text { et. al., } \\
{[11]}\end{array}$ & Yes & No & No & No \\
\hline $\begin{array}{l}\text { Belkaid } \text { et. } \\
\text { al., }[12]\end{array}$ & No & No & No & No & No \\
\hline $\begin{array}{l}\text { Tiwari } \text { et. al., } \\
{[13]}\end{array}$ & No & Yes & Yes & No & No \\
\hline $\begin{array}{l}\text { Santy } \text { et. al., } \\
{[14]}\end{array}$ & No & No & No & No & No \\
\hline $\begin{array}{l}\text { Tang } \text { et. al., } \\
{[15]}\end{array}$ & No & Yes & No & No & No \\
\hline $\begin{array}{l}\text { Lokhande } \text { et. } \\
\text { al., }[16]\end{array}$ & Yes & Yes & No & No & No \\
\hline $\begin{array}{l}\text { Mansoor } \text { et. } \\
\text { al., }[17]\end{array}$ & No & No & No & No & scaled \\
\hline $\begin{array}{l}\text { Kais } \text { et. al., } \\
{[18]}\end{array}$ & Yes & Yes & Yes & & No \\
\hline Li et. al., $[19]$ & No & Yes & Yes & Yes & No \\
\hline $\begin{array}{l}\text { Chetih } \text { et. al., } \\
{[20]}\end{array}$ & Yes & No & No & No & No \\
\hline
\end{tabular}

Table Ilb. Analysis of Parameters

\begin{tabular}{|l|l|l|l|l|l|}
\hline References & PSNR & MSE & SSIM & ROI size & Image Alignment \\
\hline $\begin{array}{l}\text { Nouredanesh } \text { et. } \\
\text { al., }[1]\end{array}$ & No & No & No & No & No \\
\hline $\begin{array}{l}\text { Zhang } \text { et. al., } \\
{[2]}\end{array}$ & No & No & No & No & No \\
\hline $\begin{array}{l}\text { Arrieta } \text { et. al., } \\
{[3]}\end{array}$ & No & No & No & No & Yes \\
\hline $\begin{array}{l}\text { Yusof } \text { et. al., } \\
{[4]}\end{array}$ & Yes & Yes & No & No & No \\
\hline Sikiö et. al., [5] & No & No & No & Yes & No \\
\hline $\begin{array}{l}\text { Scorza } \text { et. al., } \\
{[6]}\end{array}$ & No & No & No & Yes & No \\
\hline $\begin{array}{l}\text { Xiao-jun } \text { et. al., } \\
{[7]}\end{array}$ & No & No & No & No & Yes \\
\hline $\begin{array}{l}\text { Nakaram } \text { et. al., } \\
{[8]}\end{array}$ & No & No & No & No & No \\
\hline $\begin{array}{l}\text { Bérubé } \text { et. al., } \\
{[9]}\end{array}$ & No & No & No & No & No \\
\hline $\begin{array}{l}\text { Arce-Santana } \\
\text { et. al., }[10]\end{array}$ & No & No & No & No & Yes \\
\hline $\begin{array}{l}\text { Ostojić } \text { et. al., } \\
{[11]}\end{array}$ & Yes & No & Yes & No & No \\
\hline $\begin{array}{l}\text { Belkaid } \text { et. al., } \\
{[12]}\end{array}$ & No & No & No & No & No \\
\hline $\begin{array}{l}\text { Tiwari } \text { et. al., } \\
{[13]}\end{array}$ & No & Yes & Yes & No & No \\
\hline $\begin{array}{l}\text { Santy } \text { et. al., } \\
{[14]}\end{array}$ & No & No & No & No & No \\
\hline $\begin{array}{l}\text { Tang } \text { et. al., } \\
{[15]}\end{array}$ & Yes & Yes & Yes & No & No \\
\hline \begin{tabular}{l} 
Lokhande et. $[16]$ \\
\hline Mansoor et. al.,
\end{tabular} & Yes & Yes & Yes & No & Yes \\
\hline
\end{tabular}




\begin{tabular}{|l|l|l|l|l|l|}
\hline$[17]$ & & & & & \\
\hline Kais et. al., $[18]$ & Yes & Yes & No & No & No \\
\hline Li et. al., $[19]$ & No & No & No & No & No \\
\hline $\begin{array}{l}\text { Chetih } \text { et. } \text { al., } \\
{[20]}\end{array}$ & No & Yes & No & No & No \\
\hline
\end{tabular}

Table Ilc. Analysis of Parameters

\begin{tabular}{|c|c|c|c|c|c|}
\hline References & $\begin{array}{l}\text { Image } \\
\text { Similarity }\end{array}$ & $\begin{array}{l}\text { Edge } \\
\text { preservation }\end{array}$ & Encryption & Integrity & Accuracy \\
\hline $\begin{array}{l}\text { Nouredanesh } \\
\text { et.al., [1] }\end{array}$ & Yes & No & No & No & No \\
\hline $\begin{array}{l}\text { Zhang et. al., } \\
\text { [2] }\end{array}$ & No & No & No & No & No \\
\hline $\begin{array}{l}\text { Arrieta } \text { et. al., } \\
\text { [3] }\end{array}$ & Yes & No & No & No & No \\
\hline $\begin{array}{l}\text { Yusof et. al., } \\
\text { [4] }\end{array}$ & No & No & No & No & No \\
\hline $\begin{array}{l}\text { Sikiö et. al., } \\
\text { [5] }\end{array}$ & No & No & No & No & No \\
\hline $\begin{array}{l}\text { Scorza et. al., } \\
\text { [6] }\end{array}$ & No & Yes & No & No & No \\
\hline $\begin{array}{l}\text { Xiao-jun et. } \\
\text { al., [7] }\end{array}$ & Yes & No & No & No & No \\
\hline $\begin{array}{l}\text { Nakaram et. } \\
\text { al., [8] }\end{array}$ & Yes & No & No & No & Yes \\
\hline $\begin{array}{l}\text { Bérubé et. al., } \\
\text { [9] }\end{array}$ & No & No & No & No & No \\
\hline $\begin{array}{l}\text { Arce-Santana } \\
\text { et. al., }[10]\end{array}$ & No & No & No & No & No \\
\hline $\begin{array}{l}\text { Ostojić et. al., } \\
\text { [11] }\end{array}$ & Yes & Yes & No & No & No \\
\hline $\begin{array}{l}\text { Belkaid et. } \\
\text { al., [12] }\end{array}$ & No & No & Yes & Yes & No \\
\hline $\begin{array}{l}\text { Tiwari et. al., } \\
\text { [13] }\end{array}$ & Yes & No & No & No & No \\
\hline $\begin{array}{l}\text { Santy et. al., } \\
\text { [14] }\end{array}$ & No & No & No & No & No \\
\hline $\begin{array}{l}\text { Tang et. al., } \\
\text { [15] }\end{array}$ & Yes & No & No & No & Yes \\
\hline $\begin{array}{l}\text { Lokhande } e t \text {. } \\
\text { al., [16] }\end{array}$ & No & Yes & No & No & No \\
\hline $\begin{array}{l}\text { Mansoor et. } \\
\text { al., [17] }\end{array}$ & Yes & No & No & No & Yes \\
\hline $\begin{array}{l}\text { Kais et. al., } \\
{[18]}\end{array}$ & No & No & No & No & No \\
\hline Li et. al., [19] & No & No & No & No & No \\
\hline $\begin{array}{l}\text { Chetih et. al., } \\
\text { [20] }\end{array}$ & Yes & No & No & No & No \\
\hline
\end{tabular}

\section{Conclusion}

This paper gives the comparative study of testing or quality parameters for the medical image processing so that these should be used to check the correctness of an image. Testing parameter plays an important role in the medical image processing as it checks whether the image is accurate and correct by giving all information. As noise parameter is validated to check whether the image is corrupted by some noise or not. Edge- 
preservation parameter is used to validate whether the image retains its sharp edges that acts as the main part in texture smoothing of an image. Image uniformity parameter is used to check the CT numbers consistency of the images across the scan field. Image similarity is used to measure the degree of matches between image patches. Similarly many parameters should be validated to check the correctness of an image such as accuracy, integrity, PSNR, SSIM, MSE, CT number, resolution and transformation.

\section{References}

[1] M. Nouredanesh, H. R. Tizhoosh and E. Banijamali, "Gabor barcodes for medical image retrieval," 2016 IEEE International Conference on Image Processing (ICIP), Phoenix, AZ, USA, (2016), pp. 2490-2493.

[2] P. Zhang, G. Wang, Q. Huang and W. Liu, "Metrology applications of quantitative evaluation methods in CT phantom images," 2015 IET International Conference on Biomedical Image and Signal Processing (ICBISP 2015), Beijing, (2015), pp. 1-5.

[3] C. Arrieta, C. Sing-Long, S. Uribe, M. E. Andia, P. Irarrazaval and C. Tejos, "Level set segmentation with shape prior knowledge using intrinsic rotation, translation and scaling alignment," 2015 IEEE 12th International Symposium on Biomedical Imaging (ISBI), New York, NY, (2015), pp. 1568-1571.

[4] Y. W. M. Yusof, A. Saparon and N. A. Jalil, "Performance Comparison of Discrete Orthonormal STransform for the Reconstruction of Medical Images," 2015 IEEE European Modelling Symposium (EMS), Madrid, Spain, (2015), pp. 128-132.

[5] M. Sikiö et. al.,., "The effect of region of interest size on textural parameters," 2015 9th International Symposium on Image and Signal Processing and Analysis (ISPA), Zagreb, (2015), pp. 149-153.

[6] A. Scorza, G. Lupi, S. A. Sciuto, L. Battista and J. Galo, "A preliminary study on a method for objective uniformity assessment in diagnostic ultrasound imaging," 2015 IEEE International Instrumentation and Measurement Technology Conference (I2MTC) Proceedings, Pisa, (2015), pp. 1628-1633.

[7] G. Xiao-jun, H. Li-li and N. Yi, "Feature points based image registration between endoscope image and the CT image," Electric Information and Control Engineering (ICEICE), 2011 International Conference on, Wuhan, (2011), pp. 2190-2193.

[8] P. Nakaram and T. Leauhatong, "A new content-based medical image retrieval system based on wavelet transform and multidimensional Wald-Wolfowitz runs test," Biomedical Engineering International Conference (BMEiCON), 2012, Ubon Ratchathani, (2012), pp. 1-8.

[9] R. Bérubé, J. F. Pambrun and R. Noumeir, "Interoperability Testing Software for Sharing Medical Documents and Images," Internet and Web Applications and Services (ICIW), 2010 Fifth International Conference on, Barcelona, (2010), pp. 432-437.

[10] E. Arce-Santana, D. U. Campos-Delgado, A. Mejia-Rodriguez and I. Reducindo, "An innovative multimodal/multispectral image registration method for medical images based on the ExpectationMaximization algorithm," 2015 37th Annual International Conference of the IEEE Engineering in Medicine and Biology Society (EMBC), Milan, (2015), pp. 5223-5226.

[11] V. Ostojić, Đ Starčević and V. Petrović, "Recursive anisotropic diffusion denoising," in Electronics Letters, vol. 52, no. 17, (2016), pp. 1449-1451.

[12] B. M. Belkaid, L. Mourad, C. Mehdi and A. Soltane, "Secure transfer of medical images using hybrid encryption: Authentication, confidentiality, integrity," Computer Vision and Image Analysis Applications (ICCVIA), 2015 International Conference on, Sousse, (2015), pp. 1-6.

[13] V. Tiwari, P. P. Bansod and A. Kumar, "Medical imaging in heterogeneous telemedicine network," 2015 10th International Conference on Information, Communications and Signal Processing (ICICS), Singapore, (2015), pp. 1-5.

[14] A. Santy and R. Joseph, "Segmentation methods for computer aided melanoma detection," Communication Technologies (GCCT), 2015 Global Conference on, Thuckalay, (2015), pp. 490-493.

[15] Z. Tang, P. Xue, P. Yang, D. Jia and E. Dong, "An Effective Non-rigid Image Registration Method Based on Active Demons Algorithm," 2016 IEEE 29th International Symposium on Computer-Based Medical Systems (CBMS), Dublin, (2016), pp. 124-129.

[16] N. M. Lokhande, "A Matlab Based GUI for Multiframe Super Resolution Reconstruction of Different Gray Scale Images with and without Adaptive Filter," Computing Communication Control and Automation (ICCUBEA), 2015 International Conference on, Pune, (2015), pp. 668-672.

[17] A. Mansoor and M. G. Linguraru, "Generic method for intensity standardization of medical images using multiscale curvelet representation," 2016 IEEE 13th International Symposium on Biomedical Imaging (ISBI), Prague, Czech Republic, (2016), pp. 1320-1323.

[18] G. Kais, E. Noureddine and F. M. Chedly, "Comparative analysis between a variational method and wavelet method PURE-LET to remove poison noise corrupting CT images," 2016 13th International Multi-Conference on Systems, Signals \& Devices (SSD), Leipzig, (2016), pp. 287-294.

[19] D. Li, X. Li and H. Wu, "Testing of Several CT Performance Phantoms for Medical Imaging," Bioinformatics and Biomedical Engineering, (iCBBE) 2011 5th International Conference on, Wuhan, (2011), pp. 1-2. 
[20] N. Chetih and Z. Messali, "Tomographic image reconstruction using filtered back projection (FBP) and algebraic reconstruction technique (ART)," Control, Engineering \& Information Technology (CEIT), 2015 3rd International Conference on, Tlemcen, (2015), pp.1-6.

\section{Authors}

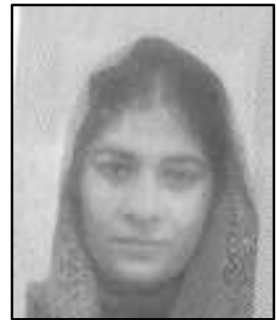

Fatima Qamar, Bachelors in Software Engineering. 
International Journal of Signal Processing, Image Processing and Pattern Recognition Vol. 10, No. 1 (2017) 